\title{
Analyzing Distributed Whiteboard Interactions
}

\author{
Lutz Gericke, Raja Gumienny, Christoph Meinel \\ Hasso Plattner Institute Potsdam \\ Prof. Dr. Helmert Str. 2-3, Potsdam, Germany \\ Email: \{firstname.lastname\}@ hpi.uni-potsdam.de
}

\begin{abstract}
We present the digital whiteboard system TeleBoard, which automatically captures all interactions made on the all-digital whiteboard and thus offers possibilities for a fast interpretation of usage characteristics. Analyzing team work at whiteboards is a time-consuming and error-prone process if manual interpretation techniques are applied. In a case study, we demonstrate how to conduct and analyze whiteboard experiments with the help of our system. The study investigates the role of video compared to an audio-only connection for distributed work settings. With the simplified analysis of communication data, we can prove that the video teams were more active than the audio teams and the distribution of whiteboard interaction between team members was more balanced. In this way, automatic analyses can not only support manual observations and codings, but also give insights that cannot be achieved with other systems.
\end{abstract}

Index Terms - whiteboard, remote collaboration, team analysis

\section{INTRODUCTION}

Whiteboards are essential instruments when design teams are working together. In many companies, they serve as the main tool for collecting ideas, visualizing insights, and gathering tasks and concepts [22]. For many everyday tasks, a lot of team communication is supported by whiteboards, because of their ease of use and instant availability. In colocated working environments, people mostly use traditional whiteboards [10], [22]. Problems arise when teams have to work in a distributed environment and they still want to keep their usual way of working at a whiteboard.

Although whiteboard systems - mostly image or video based - have been in the research focus for over twenty years (e.g. [13], [16], [18], [23], [25]), distributed teams only rarely use these systems in corporate or research environments [10]. A reason may be that it is still hard to evaluate and analyze whiteboard use in order to find out how people interact with them and which difficulties they have. Digital systems always work differently from their analog counterparts and only if we understand their utilization and the main obstacles in using them, can we design systems people are willing to adopt. With the mentioned problems in mind, our main goals that we describe in this article are:

1) Supporting people who are working with a completely digital whiteboard solution such as Tele-Board

2) Fast and convenient analysis of design team activity by researchers

Both points are mutually dependent. That means, an analysis of design activity needs tools that can be properly used by design teams - especially for distributed settings. In order to develop these tools, research has to be done on how design teams work and how they use the given tools. In this paper, we focus on analyzing design teams using Tele-Board as a system for automatic analysis. The results of the case study itself lead to implications that help us to improve the system setup.

We present an all-digital whiteboard system for distributed work, which includes automatic analysis functions in order to ease the evaluation of interaction on the board. The TeleBoard system combines a full-screen video conference with a synchronous transparent whiteboard overlay. This setup enables regionally separated team members to simultaneously manipulate artifacts while seeing each other's gestures and facial expressions.

In a case study, we tested how adding video to the shared whiteboard improved the interaction experience in contrast to an audio-only setup. While we ascertained that the video teams were faster and more active during their work on the whiteboard, we could also show that the automatic analysis largely corresponds with manual video codings and therefore reduces manual analysis efforts.

In addition to a simplified analysis, it is also possible to interpret whiteboard work without positioning cameras in workplaces and thereby creating a situation of surveillance [2]. Researchers can easily work with the collected data at any point in time and the consistent digitalization of all data gives the possibility to investigate aspects of whiteboard work that have not been focused on before.

\section{RELATED WORK}

Capturing and analyzing design activity has been a field of major interest for a long time, but collecting data of design teams in realistic work settings is difficult [5], [14], especially if design teams are regionally separated across different locations. The Design Observatory and the iLoft project are examples of where design spaces are equipped with collaboration and information technology in order to attract design teams to work there [4], [14]. Video and audio recording equipment gives researchers the possibility to examine and analyze design activity in this space. However, the analysis of video and audio material is mostly done manually and thus is a very time consuming process.

There is already a variety of research projects that focus on capturing and analyzing team work, though the domain and collaboration settings vary. Richter et al. for example, 
created the TeamSpace system, which captures meetings of distributed workgroups [19]. However, it is adapted to standard meeting behavior and does not match the irregular episodic nature of design work [12]. In recent research work, Uflacker et al. do analyze design teams' work, though they focus their analysis on e-mails, wikis and shared folders [27]. From our point of view it is also necessary to capture the actual work at whiteboards where people visualize their ideas and sketch prototypes. The projects Designer's Outpost [13] and WorkspaceNavigator [12] capture designers' work at whiteboards and give users the possibility to scroll through a timeline of whiteboard states. However, the whiteboard states are captured as images, which need to be interpreted by humans. Further (automatic) analyses of whiteboard interactions - such as sketching activity - cannot be done, as the data does not provide this fine-grained access. In the Coral project, Minnemann et al. created a recording system for the digital whiteboard software Tivoli [15]. The whiteboard interaction data and other multimedia streams are saved for later access of the team's work. This provides the possibility for asynchronous work. How well synchronous design work across distances is also supported, is not evident.

The first tools to support creative collaboration of spatially separated teams were VideoDraw [26], VideoWhiteboard [25], and Clearboard [11]. VideoWhiteboard especially fits the requirements of collaborative teamwork, as it transfers whiteboard content with the help of a rear projection to the whiteboard of a remote person. Additionally, a shadow image of the remote person's upper body is transferred to see gestures of the partner. Seeing only the shadow and not a real video of the other person is one limitation which Tang et al. point out themselves [25]. Even more important is the missing functionality of manipulating the other person's drawings and working on the same artifacts. This drawback also arises with the Clearboard and Facetop [21] system, although it is possible to see a real image of the other person rather than only a shadow. Other projects also included shadows of the remote person combined with transient ink to convey deictic gestures [13], or inserted a video of the remote person's arms [23]. There are various other digital whiteboard systems, which were developed in the past twenty years e.g. Flatland [16] or Tivoli [18], but most of them have been developed for co-located use only and do not focus on remote setups and therefore do not involve video functionalities.

In order to analyze distributed whiteboard interactions holistically and efficiently, i.e. as time-saving as possible, we developed a digital whiteboard system infrastructure, which provides these functionalities.

\section{TElE-BOARD - Distributed WhiteboARD INTERACTION}

Today, whiteboards are ubiquitously used in all offices in companies of all industries as well as in universities and schools. People meet for a brainstorming to discuss a design or simply to collect some ideas. Problems arise if teams are distributed all over the world and cannot meet easily. Although digital whiteboards are implemented in many online-meeting applications, they often do not fit people's needs and are not used frequently [10].

Tele-Board is meant to be a solution for people who often work at whiteboards, using huge amounts of sticky notes and other tangible tools - such as paper and pens - to visualize their ideas and designs [3]. It is our goal to support this way of working also for geographically dispersed teams, that is to say a somehow digital solution, which retains the working modes of the analog world as closely as possible [8]. Additionally, the system shall have all advantages of a digital solution, as for example saving whiteboard states and continuing at another place of work. Therefore, Tele-Board uses a paradigm of message exchange and capturing, which enables synchronous as well as asynchronous interactions [7] seamlessly integrated into one system. In contrast to other solutions (e.g. [2], [9]), we do not store images but the communication flow itself. In the following, we describe the system's architecture and how it enables natural design work as well as an uncomplicated analysis of past design activities.

\section{A. Supporting synchronous and asynchronous work}

The user interface of the Tele-Board whiteboard client focuses on the traditional (analog) whiteboard metaphor: scribbling on the board and wiping off the scribbles are the elementary functions. Furthermore, the Tele-Board system gives the possibility of writing sticky notes with different devices (e.g. Tablet PC and iPad, smartphone, digital pen, or keyboard). By creating the sticky notes digitally instead of capturing paper notes, media gaps and capturing time as in The Designer's Outpost [13] can be omitted. For a remote setup, it is possible to open multiple instances of the same whiteboard. All whiteboard elements and actions are synchronized between the whiteboard instances, enabling every user to always see the team members' actions and manipulate all sticky notes and drawings, no matter who created them. This is a major advantage compared to Clearboard [11] and VideoWhiteboard [25] where you can only manipulate your own whiteboard marks.

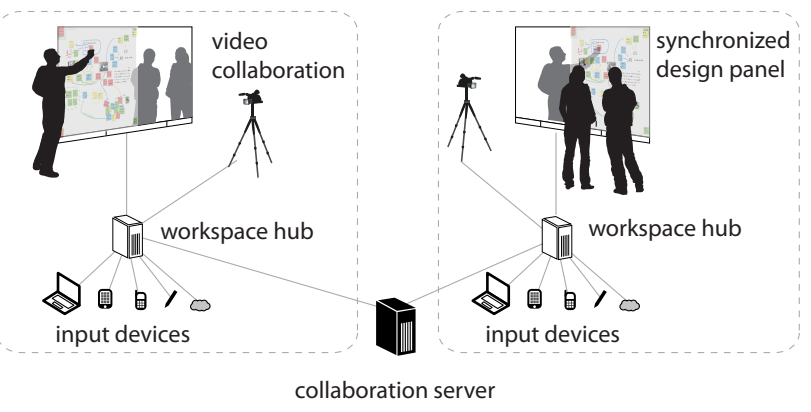

Fig. 1. Tele-Board general system infrastructure, exemplary for two workspaces

The main interface for user interaction is the whiteboard client. This client is accessible via Java Webstart from a 
web portal and can be opened on any computer running a Java virtual machine. In order to have the look and feel of traditional whiteboards, it should be started on large scale digital whiteboard hardware as for example a SMART Board ${ }^{1}$. Nevertheless, the software also runs on standard computers or Tablet PCs. During development, we collected feedback from a variety of different users in order to make the usage more natural. A crucial part of the natural feeling of interactions is the integration of video conferencing equipment. We experimented with two camera setups (see figure 2). One of them (left) uses a 45 degree camera angle towards the edge of the whiteboard, capturing the foreshortened whiteboard and the people in front of it. This way, people can face both the whiteboard and the camera at the same time and have the feeling of standing next to each other. In the second setup (right), the camera stands orthogonal towards the center of the board. This setup has two advantages: people have double the whiteboard space to work on and pointing on certain areas on the board can be seen more accurately on the remote side. Based on these advantages, we used the orthogonal video setup during the case study experiment (figure 1 shows the angular video setup).
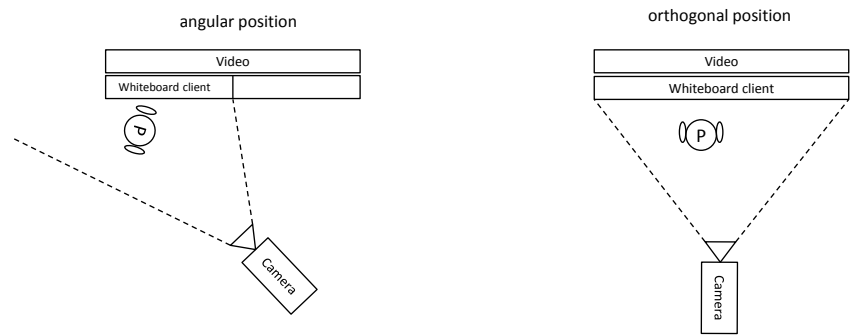

Fig. 2. Different camera setups - left: angular position, full screen video with half screen translucent whiteboard overlay; right: full screen video and full screen translucent whiteboard overlay

Equally as important as synchronous whiteboard work is the possibility to work asynchronously, i.e. at different points in time. In a typical asynchronous interaction scenario, a user wants to see how the content of one or more whiteboards was generated and view different states of the creation process. Therefore, we developed the Tele-Board history browser, which offers a timeline-view of all actions made on the board. Through a web portal people can retrace what has happened - they only need a web browser, no matter where they are.

The history functions and possibilities of whiteboard analyses are closely connected as they both rely on the transmission and storage of communication data. In the following, we describe the communication infrastructure and message capturing of the Tele-Board system.

\section{B. Communication flow and message capturing}

Figure 1 shows an outline of the general system architecture and communication infrastructure: A central collaboration

\footnotetext{
${ }^{1}$ http://www.smarttech.com/
}

server connects all workspace hubs and routes communication messages between them. A workspace hub is located at each workspace location and all input devices at this location are logically connected to it (not necessarily physically). The centralized architecture provides the opportunity to capture all communication data that is exchanged between the clients. Archiving this data offers the chance to reconstruct the communication afterwards, including every single detail as it has been transmitted in the original message flow. The communication itself is realized via XMPP ${ }^{2}$ - a protocol developed for instant messaging. We extended the standard XML messages in order to transmit whiteboard content modifications, such as for drawing or the erasing of scribbles.

In order to capture whiteboard states and analyze them, a history archive is necessary. Basically this is a database containing the complete message flow of a whiteboard session, including all data that is needed to reconstruct every moment of the whiteboard interaction. To fill the database with content, the message flow has to be intercepted and stored. As we are using the open standard XMPP, a number of open source server software is available. For our setup we decided on Openfire ${ }^{3}$ as the XMPP server, which is easily extensible via plug-ins. The TeleBoardServerBuddy plugin processes and stores every whiteboard communication message.

The system's work units are organized in Projects and Panels. A Panel $p$ describes the sequence of events $e_{n}$ executed on one whiteboard in temporal order of these events ( $p=$ $\left.\left(e_{1}, e_{2}, e_{3}, \ldots\right)\right)$. An event is a tuple of attributes describing which action has happened where, by whom, and when. Each event has an operation code, which can be NEW, CHANGE or DELETE to describe the event type. A Project pro is the collection of multiple Panels $\left(\right.$ pro $\left.=\left\{p_{1}, p_{2}, \ldots\right\}\right)$ in order to configure them with access rights to edit/view/delete. To give an idea of the data format, table I shows the representation of scribble and sticky note event messages in the database. For a detailed description how data is stored within the system and how typical asynchronous interaction within teams is realized, see [7].

TABLE I

EXAMPLE DATABASE ENTRIES FOR SCRIBBLE PATH AND STICKY NOTE EVENTS

\begin{tabular}{|c|c|c|c|c|c|c|}
\hline id & create_time & panelid & obj_data & opcode & username & object_type \\
\hline $2056: 0$ & $\begin{array}{l}2010-09-21 \\
11: 48: 09\end{array}$ & 1 & $\begin{array}{l}\text { <path } d=" M 432.0 \\
\mathrm{~L} 432.0 \ldots\end{array}$ & NEW & $\begin{array}{l}\text { markus } \\
\end{array}$ & path \\
\hline $2056: 0$ & $\begin{array}{l}2010-09-21 \\
11: 48: 12\end{array}$ & 1 & $\begin{array}{l}<\text { path } d=" M 432.0 \\
\mathrm{~L} 432.0 \ldots\end{array}$ & CHANGE & markus & path \\
\hline 2056:1 & $\begin{array}{l}2010-09-21 \\
11: 50: 02\end{array}$ & 1 & $\begin{array}{l}<\text { postit sizex }=" 90 " \\
\ldots\end{array}$ & NEW & markus & postit \\
\hline 2056:1 & $\begin{array}{l}\text { 2010-09-21 } \\
11: 50: 04\end{array}$ & 1 & $\begin{array}{l}<\text { postit sizex }=" 90 " \\
\ldots\end{array}$ & CHANGE & markus & postit \\
\hline$\cdots$ & $\ldots$ & $\ldots$ & $\ldots$ & $\ldots$ & $\ldots$ & $\cdots$ \\
\hline
\end{tabular}

In order to show that the chosen architecture is appropriate for research on team interaction, we conducted a case study, which is shown in the following section.

\footnotetext{
${ }^{2}$ Extensible Messaging and Presence Protocol, RFC 3920-3923

${ }^{3}$ http://www.igniterealtime.org/projects/openfire/
} 


\section{Analyzing Design Activity With Tele-BoARd - A CASE STUdy}

With the help of a case study we wanted to test if our system is able to run large parts of an experiment analysis automatically. Our goal was to gather insights on which data must be captured, in order to get meaningful results about team interaction. Typically, when observing teams and analyzing their performance, researchers get their data from codings of video camera recordings. We wanted to investigate whether the data that was gathered with our solution was as meaningful as manually gathered data. With equal or better quality of automatic analyses, it could be shown that manual codings can be (partly) replaced.

For the exemplary study we chose to test the role of video for remote collaboration. Various researchers have explored the importance of video compared to audio-only systems, though setups and results varied a lot in factors that involved people, size and orientation of video, quality of audio and video and the tasks the people had to do (e.g. [1], [17], [28]). We tested the Tele-Board system with regard to the advantages of a full screen video behind the whiteboard surface compared to an audio-only connection. In both conditions participants worked with the synchronized digital whiteboard software.

\section{A. Case Study Design}

To create a task that cannot be solved easily and requires users to concentrate a lot, we asked people to solve a "logic grid puzzle". Their task was to jointly investigate a car theft with the help of ten facts written on sticky notes (e.g. "person A did not steal the car at person B's house"). A team of two persons worked in a remote location setup, which was connected by two synchronized whiteboards. Two conditions were tested:

- the team communicates only via audio (see figure 3) and

- the team communicates via audio and sees a video of the other person in full screen on the whiteboard (see figure 4)

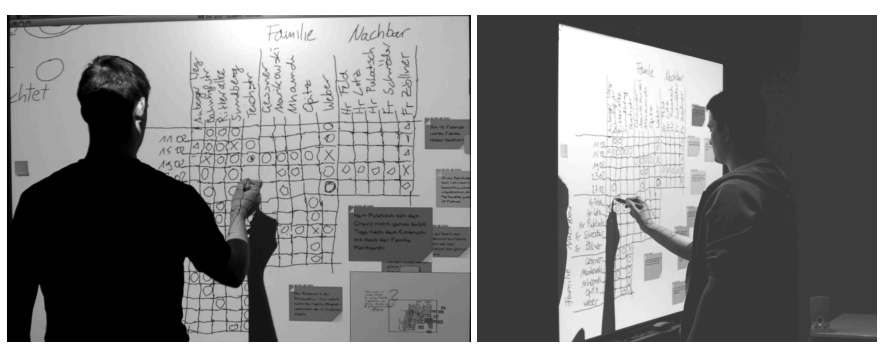

Fig. 3. Remote study participants in the audio-only condition. Users cannot see which part of the grid their partners are currently editing

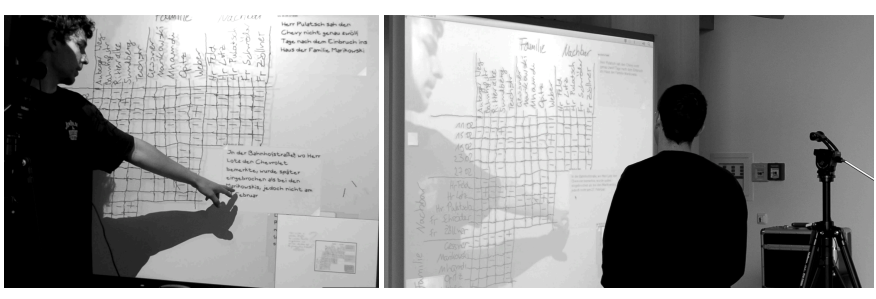

Fig. 4. Remote study participants in the video condition. Deictic gestures are easily visible for the remote partner

Based on previous research and our experience with the system we had the following hypotheses:

1) With a video, people are more efficient, i.e. solve the task faster.

2) With a video, people enjoy the task more, establish more common ground, more team spirit, and work together more closely.

Ten pairs of undergraduate computer science students from the same university participated in the study. They were all male, between 19 and 24 years old, and partners were selected who did not know each other before the study. We used a between-subjects design instead of a within-subjects design because logic grid puzzles take a long time $(30-60 \mathrm{~min})$ and learning effects are very intensive. The participants will know each other better after repeated tests, get more used to the task and each other. Moreover, subjects do not know both conditions and therefore will not vote for the setup they consider "our solution".

Before every test, the two participants met at one of the two locations [20]. An instructor read out loud the context of the car theft story and told them that they should solve the task together on a shared whiteboard. Afterwards the instructor showed the required functions of the SMARTBoard ${ }^{4}$ (touch, pen and eraser input) and the Tele-Board software (drawing, moving the whiteboard surface, resizing and changing the color of sticky notes). The participants tried out the functions until they felt confident using the system (not longer than 5 minutes each). After one of the participants went over to the other location and the participants felt ready to start, the stop watch was started. The task ended when the participants correctly solved the puzzle or after approximately one hour when it was foreseeable that they would not solve the puzzle correctly. In the end, participants completed a questionnaire on their experiences.

1) Measures: We used a questionnaire to assess the constructs "joy of use", "common ground", "team spirit" and "well-being" (with 7-point Likert scales). Each of the constructs was assessed via multiple indicator variables (items). The items for each construct were tested regarding correlation and afterwards the mean value of all items of a construct was calculated. In total, the questionnaire contained 35 items.

Performance was assessed in terms of task completion time. We also collected video recordings of all experiments and

\footnotetext{
${ }^{4}$ http://www.smarttech.com/
} 
analyzed them with regard to noticeable utterances and actions. We measured the following events: whiteboard activity person 1 (interval), whiteboard activity person 2 (interval), reference gesture person 1 (marker), reference gesture person 2 (marker), verbal utterance person 1 (interval), verbal utterance person 2 (interval). Additionally, we used the whiteboard history data, which is logged automatically by our system for measuring whiteboard activities.

An overview of the measures can be seen in table II, where we present how to take the measurements with the help of different observation methodologies. A major difference between the three methodologies is the amount of time spent. Manual observations, questionnaires, as well as video codings take hours of time, whereas automatic analyses are available instantly.

TABLE II

ANALYSIS METHODOLOGIES FOR THE EVALUATION OF DESIGN ACTIVITY (N.I.Y. = NOT IMPLEMENTED YET $)$

\begin{tabular}{l|l|c|l} 
measure & $\begin{array}{l}\text { manual, } \\
\text { questionnaire }\end{array}$ & video coding & $\begin{array}{l}\text { automatic } \\
\text { analysis }\end{array}$ \\
\hline \hline social measures, emotions & yes & partly & no \\
\hline whiteboard activity & no & partly & yes \\
\hline referencing, gestures & no & yes & n.i.y. \\
\hline verbal interaction & no & yes & n.i.y. \\
\hline performance, time-consumption & yes & yes & yes \\
\hline
\end{tabular}

\section{B. Manual analysis of design activity}

This section shows the manual steps of analyzing design team activity. The logic grid puzzle experiment shall be a case study for showing how comparable whiteboard experiments can be conducted and evaluated. To evaluate the experiment, we started with analyzing the post-test questionnaire results and video coding data. Although the number of participants was too small to derive general conclusions, we could find very interesting insights and trends concerning the system's usage. As we tested ten groups and videotaped each location separately, we recorded over 20 hours of video.

1) General user feedback: In general, participants were satisfied with their respective condition. In post-test interviews all members of the audio teams stated that the audio channel was important and sufficient for their communication. Some of them said that a pointing device would have been nice to show their partners which part of the whiteboard they were talking about. One person said it would have been "nicer" with a video. The majority of the participants of the video condition said that they liked the video and that it helped a lot to reference what they were talking about. This corresponds to findings of other researchers (e.g. [6], [24]).

2) Questionnaire analysis: In general, participants rated questions regarding joy of use quite high and in the video condition even slightly higher (1.82 vs. 2.34 with $1=$ very positive and $7=$ very negative) (see figure 5 ). In the video condition, users also stated that they could better understand what their partner wanted to tell them and vice versa (2.55 vs. 2.95). The analysis of questions regarding team spirit did not show any major tendencies towards one condition (2.96 video vs. 3.10 audio). Surprisingly, users rated their well-being a little higher in the audio condition (3.12 video vs. 2.88 audio).

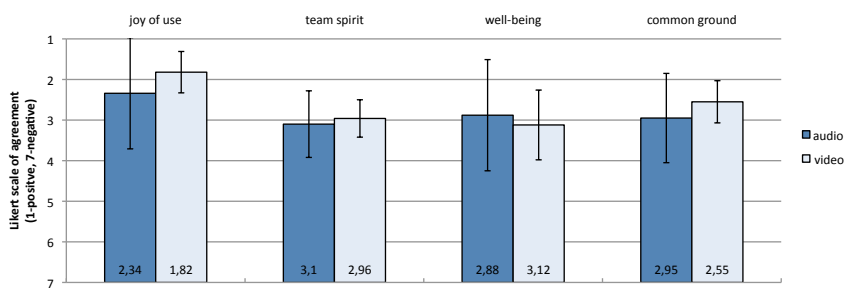

Fig. 5. Questionaire results comparing joy of use, team spirit, well-being, and common ground for audio and video condition

3) Video coding: During the test, we found that audio teams sometimes had some difficulties finding out what their partners were talking about. Though they knew that their partners could not see their gestures they often pointed at certain positions in the grid. With every audio team, dialogs such as "I don't know where you put the last crossmark" "Here, at the lower left part of the grid" or "Are you doing something?" - "No I'm just thinking" occurred. However, coordination problems happened less often than we expected (about twice per team) and they heavily depended on the respective team. All video teams made use of the possibility to directly point at certain cells in the grid combined with saying "here" instead of explaining which part of the grid they were talking about. This obviously helped to establish a common ground, and when one person pointed at one cell the other person set the whiteboard mark [24]. Nevertheless, the amount of referencing also differed between teams. Some worked together very closely and referenced a lot while others worked more individually.
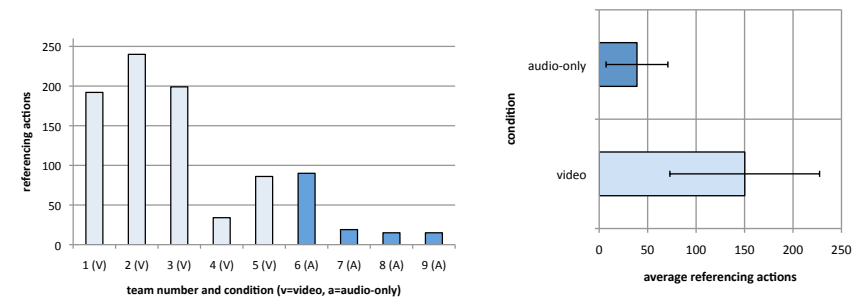

Fig. 6. Amount of referencing actions; left: per team, right: average per condition

Part of the video-coding were the markers of spatial referencing gestures made by the participants. Often, pointing gestures are supported by spoken instructions or remarks of the participant. Figure 6 shows these observations compared between both conditions. People in the video condition did by far more pointing gestures than the audio-only teams did. Additionally, people could be more self-confident when drawing on the board because they saw that the partner did not interfere with their activity. Even though the audio teams were aware of the fact that the team partner could not see any 
gestures, it is interesting to see that people naturally did these gestures as well.

\section{Automatic analysis using Tele-Board history-based statis-} tics

In this section, we show the possibilities of an analysis based on the whiteboard history data. For our automatic analysis of team interaction and performance, we captured 21674 whiteboard element events during the experiments. As shown in section III this data is stored in a database giving a row-by-row representation of the whiteboard actions, such as moving a sticky note or drawing a line on the whiteboard surface.

1) Team performance: The first hypothesis on differences in team performance between the conditions, can be answered by calculating the difference between the first and the last whiteboard event. Figure 7 shows the task completion time of each team. Overall, a difference between video and audio teams is visible. The average values show a significance towards our hypothesis that the audio-only teams needed more time than the teams in the video condition. A two-sided t-test with a p-value of 0.017 confirms this.
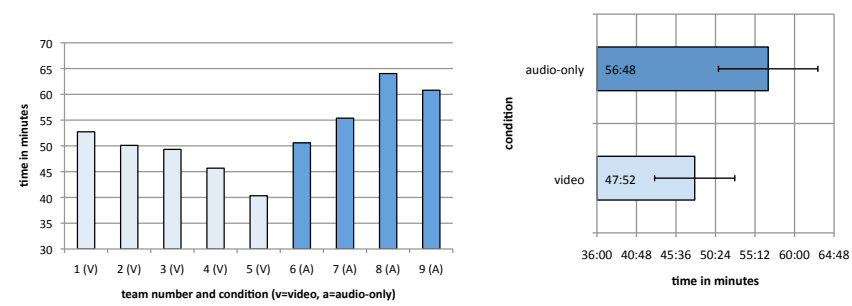

Fig. 7. Task completion time; left: per team, right: average task completion time per condition

2) Whiteboard activity: Additionally, we could not only see that video teams were faster on average, but they were also more active. Looking at the ratio $r=$ time $_{\text {active }} /$ time $_{\text {all }}$, i.e. the time when the participants were interacting with the whiteboard divided by the overall task completion time, there is also a significant difference (p-value of 0.042) between both conditions. Figure 8 shows the activity ratio for each team and the mean values for both conditions.

Obviously, people tend to be more active when they see an image of their remote partner. Especially with the logic grid task, people could largely benefit from the video. They were pointing at certain areas on the whiteboard and then discussed how to add their conclusions to the puzzle. In the video condition, we could also see that people are more effective in their collaboration as we could find less time intervals without interactions. Nonproductive time-intervals are larger within the audio-only condition (see figure 10).

3) Team member activity distribution: We also examined the deviation of activities between the two partners, because we wanted to know whether the conditions have influence on an equal distribution of whiteboard interactions (see figure 9). One can see that the whiteboard interactions for some
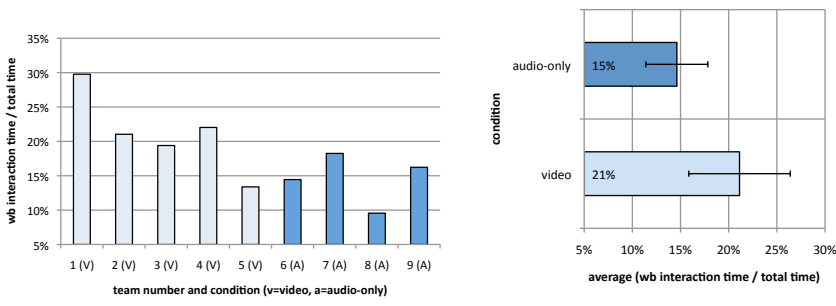

Fig. 8. Ratio between activity on the whiteboard and the overall task completion time; left: per team, right: average per condition

teams are distributed quite evenly, e.g. teams 1 (V), 9 (A), whereas in other teams one person did over $80 \%$ of all whiteboard actions (teams $3(\mathrm{~V}), 6(\mathrm{~A})$ and $8(\mathrm{~A})$ ). We assumed that interactions are distributed more evenly in the video condition as we hypothesized that these teams were working together more closely. Although we could not find significant differences between the conditions, we still regard this analysis of interaction distribution as an important way to evaluate team work. Obviously, there are major differences between the teams and these correspond with our subjective evaluation of team work and team spirit after the tests. It could be interesting to study the distribution of interactions while manipulating other variables, as team spirit and group cohesion not only depend on media condition but several other factors [29] as well.

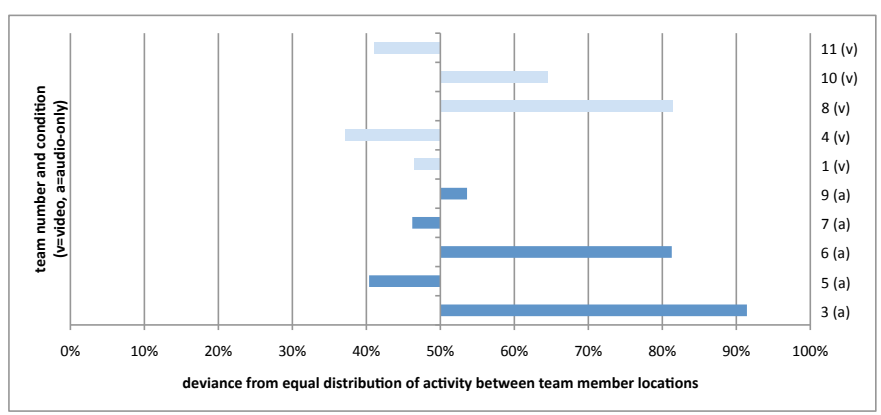

Fig. 9. Deviation of whiteboard activities between the two partners

\section{Validation of Tele-Board history data with manual video codings}

Often, automatically analyzed observation data cannot fully represent the activity that really took place in the experiments. In order to validate that the recorded whiteboard communication data can give a sufficient representation of activity on the whiteboard, we assigned manual video codings to the experiment recordings. As the coding instrument, people were instructed to use $\mathrm{VCode}^{5}$, an open-source video annotation tool. Twelve different measures were coded, such as whiteboard actions on both sides (as intervals), as well as spatial referencing gestures (as event markers).

\footnotetext{
${ }^{5} \mathrm{http} / / /$ social.cs.uiuc.edu/projects/vcode.html
} 
The two coders for each video had an agreement of over $90 \%$. A comparison of whiteboard history data aligned with video coded events, shows a high degree of similarity between coding and history data. The high overlapping (also over $90 \%$ ) between both methods (manual video coding and automatic history analysis) was computed statistically, but can be better seen in figure 10. As proven by the four teams, the difference between video coding and an automatic analysis with our system is only marginal.

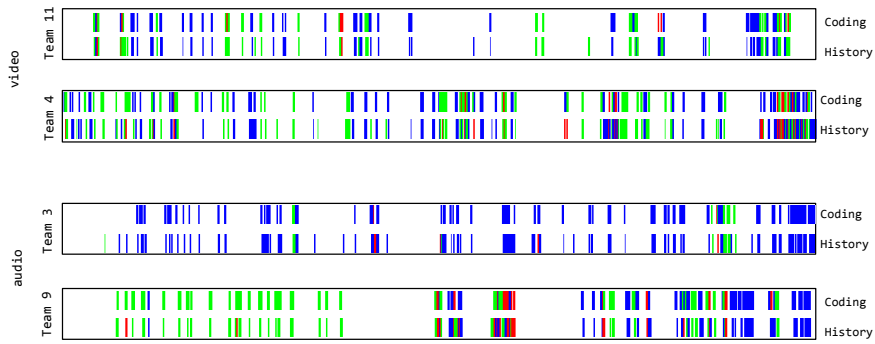

Fig. 10. Timeline comparison of video coding data vs. recorded history data; colors: green $=$ first person active, blue $=$ second person active, red=both persons active simultaneously

\section{E. Advantages and limitations of an automatic analysis with Tele-Board}

Summing up the findings of the case study, the whiteboard history data and video coding data came to the same conclusion: although the video teams were more active and created more whiteboard element events they solved the task faster than the teams in the audio condition.

The combination of traditional methods such as questionnaires and automatically derived information from the TeleBoard history can be a powerful tool to find linkages between these different measures. For example, we can find hints as to why people rated the "joy of use" criterion higher in the video condition. Disregarding the question, whether the video condition was more effective, the values show that it was: faster, more active in terms of whiteboard activity, and invited many more pointing gestures.

One can see that the method of recording the complete communication channel can reveal unforeseen interrelations. Certain characteristics that are found in the questionnaire results can be analyzed in more detailed with the help of the history archive. This way, researchers can save hours of video coding time and receive more accurate results.

\section{CONCLUSiOn AND OUTLOOK}

We showed a concept for design team observation and analysis, illustrated by a prototypical implementation. The idea of recording original communication data has shown to be valuable when analyzing team behavior. The exemplary case study "logic grid puzzle" gave an impression of how team performance analysis can be conducted in an all-digital setting using the Tele-Board whiteboard system.

As a conclusion of this exemplary case study we found out: a video-enabled connection makes a difference. This difference was shown to be significant, even though there was only a small number of participants. Video-teams were faster, more active, and used their time more effectively.

As a consequence for traditional design team analysis, we conclude that it is less time-consuming to analyze teams with a solution such as the one we presented here. An automatic analysis cannot completely replace manual codings, but there are large overlapping areas. Observers do not have to concern themselves with key figures, such as task completion time or events per minute. Researchers can fully focus on analyzing and interpreting the data. On the other hand, computersupported team observation is more accurate, because manual video codings with potential errors are avoided.

The history archive offers substantial advantages to traditional whiteboards - not only for researchers but for whiteboard users, too. As we mentioned earlier, people can browse through their past whiteboard sessions in order to recap what they did and get a better understanding of their own and the team's work. A common ground of knowledge and understanding - especially within distributed teams - can be better established. Generally, there is a large need in all kinds of businesses to archive communication data and make use of it. One example: assuming that the Tele-Board whiteboard client was used by a consulting agency to sketch business processes for a customer. When iterating on the developed models, multiple persons' perspectives are taken into account. In a final stage, when it comes to retracing who said what regarding a certain decision in the model, it might be important to find exactly that spot in the history. Our system could easily help here, whereas it was almost impossible to find this decision point before. Additionally, teams can go on working at certain points in the past and start a parallel branch of the whiteboard content.

Through interviews we found that people do not want to browse through hours of archived material, no matter if it is video, audio or a whiteboard history. We gather more and more insights on how to automatically "understand" what has happened in the past, in order to show the most important points of the respective sessions. Identifying temporal phases of meaningful interactions is a promising approach, which we will address in the near future. Figure 10 already points to this kinds of analyses. One can see that people are initially very active. Later, they have longer phases of no interaction. In the experiments we observed this pattern when participants reached the turning point from gathering facts to reasoning on the facts.

Besides the analyses of data we presented in the case study, the history archive can be used for various other kinds of data plots. For example sticky note traces (see figure 11), where all sticky note movement operations are visible. When a sticky note is dragged on the whiteboard, a blue line represents the movement from the starting point to the end point. The data shown here is also taken from the logic grid puzzle experiment.

In the video condition, participants obviously moved the sticky notes more extensively than the audio-only teams. This coincides with our other findings that the video teams were 

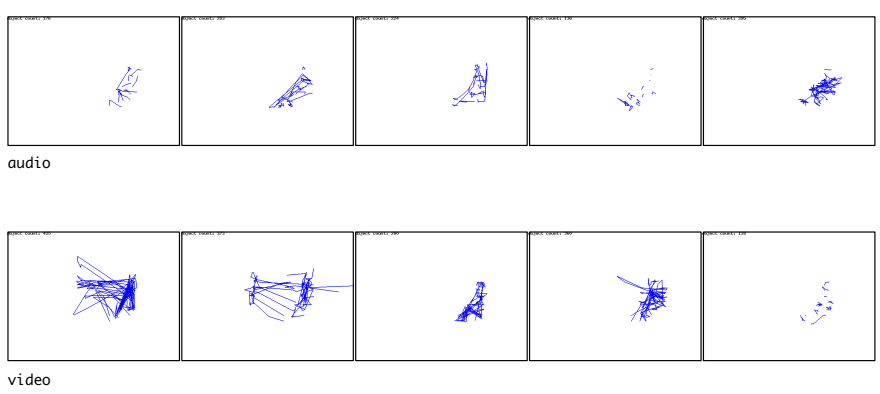

Fig. 11. Sticky note movement traces. Blue lines outline the movement path of a sticky note during its lifetime

more active. We assume that people are more actively working with the whiteboard in the video condition, because they feel more confident using it. Seeing the partners gestures and interactions prevents editing conflicts and gives the feeling of more natural discussions.

Creating and filling a history archive with communication and interaction data is very data intensive, because every single operation is captured and stored. But this strategy is extremely valuable, because it gives the opportunity to run various kinds of analyses on the data - even on aspects that have not been in focus before an experiment. This concept and the implementation within the Tele-Board system show high potential for further extensions and activities.

\section{ACKNOWLEDGMENT}

We would like to thank the HPI-Stanford Design Thinking Research Program for funding and supporting the project.

\section{REFERENCES}

[1] N. Bos, J. S. Olson, D. Gergle, G. M. Olson, and Z. Wright. Effects of four computer-mediated communications channels on trust development. In CHI '02: Proceedings of the SIGCHI conference on Human factors in computing systems, pages 135-140, 2002.

[2] S. Branham, G. Golovchinsky, S. Carter, and J. T. Biehl. Let's go from the whiteboard: supporting transitions in work through whiteboard capture and reuse. In CHI '10: Proceedings of the SIGCHI conference on Human factors in computing systems, 2010.

[3] T. Brown. Design Thinking. Harvard Business Review, (June):84-92, 2008.

[4] K. Carrizosa, O. Eris, A. Milne, and A. Mabogunje. Building the Design Observatory: a Core Instrument for Design Research. In International Design Conference, 2002.

[5] N. Cross, H. Christiaans, and K. Dorst. Analysing Design Activity. WileyBlackwell, 1996.

[6] S. R. Fussell, R. E. Kraut, and J. Siegel. Coordination of communication: effects of shared visual context on collaborative work. In CSCW '00: Proceedings of the 2000 ACM conference on Computer supported cooperative work, pages 21-30, New York, NY, USA, 2000. ACM.

[7] L. Gericke, R. Gumienny, and C. Meinel. Message Capturing as a Paradigm for Asynchronous Digital Whiteboard Interaction. In 6th International ICST Conference on Collaborative Computing: Networking, Applications and Worksharing, 2010.

[8] R. Gumienny, L. Gericke, M. Quasthoff, C. Willems, and C. Meinel. Tele-Board : Enabling Efficient Collaboration In Digital Design Spaces. In CSCWD '11, 2011.

[9] L.-w. He, Z. Liu, and Z. Zhang. Why take notes? Use the whiteboard capture system. In IEEE International Conference on Acoustics, Speech, and Signal Processing, 2003 (ICASSP '03)., pages V-776-9. Microsoft Research, IEEE, 2003.
[10] E. M. Huang, E. D. Mynatt, D. M. Russell, and A. E. Sue. Secrets to Success and Fatal Flaws: The Design of Large-Display Groupware. IEEE Computer Graphics and Applications, 26(1):10-17, 2006.

[11] H. Ishii and M. Kobayashi. ClearBoard: a seamless medium for shared drawing and conversation with eye contact. In $\mathrm{CHI}$ '92: Proceedings of the SIGCHI conference on Human factors in computing systems, pages 525-532. ACM New York, 1992.

[12] W. Ju, A. Ionescu, L. Neeley, and T. Winograd. Where the wild things work: capturing shared physical design workspaces. In Proceedings of the 2004 ACM conference on Computer supported cooperative work CSCW '04, pages 533-541, New York, New York, USA, Nov. 2004. ACM Press.

[13] S. R. Klemmer, K. Everitt, and J. Landay. Integrating Physical and Digital Interactions on Walls for Fluid Design Collaboration. HumanComputer Interaction, 23(2):138-213, Apr. 2008.

[14] A. Milne and T. Winograd. The iLoft project: A technologically advanced collaborative design workspace as research instrument. In Proceedings of the 13th Annual Intenational Conference on Engineering Design (ICED03), pages 1-10. Citeseer, 2003.

[15] S. Minneman, S. Harrison, B. Janssen, G. Kurtenbach, T. Moran, I. Smith, and B. van Melle. A confederation of tools for capturing and accessing collaborative activity. In Proceedings of the third ACM international conference on Multimedia - MULTIMEDIA '95, pages 523-534, New York, New York, USA, Jan. 1995. ACM Press.

[16] E. D. Mynatt, T. Igarashi, W. K. Edwards, and A. LaMarca. Flatland: new dimensions in office whiteboards. In CHI '99: extended abstracts on Human factors in computing systems, pages 346-353, 1999.

[17] J. S. Olson, G. M. Olson, and D. K. Meader. Face-to-face group work compared to remote group work with and without video. In K. Finn, A. Sellen, and S. Wilbur, editors, Video Mediated Communication, pages 157-172, Hillsdale, NJ, 1997. Lawrence Erlbaum Associates.

[18] E. R. n. Pedersen, K. McCall, T. P. Moran, and F. G. Halasz. Tivoli: an electronic whiteboard for informal workgroup meetings. In Proc INTERACT '93 and CHI '93, ACM Press, pages 391-398, 1993.

[19] H. Richter, G. Abowd, W. Geyer, L. Fuchs, S. Daijavad, and S. Poltrock. Integrating Meeting Capture within a Collaborative Team Environment. In Proceedings of Ubiquitous Computing 2001, volume 2201 of Lecture Notes in Computer Science, pages 123-138, Berlin, Heidelberg, Oct. 2001. Springer Berlin Heidelberg.

[20] E. Rocco. Trust breaks down in electronic contexts but can be repaired by some initial face-to-face contact. In Proceedings of the SIGCHI conference on Human factors in computing systems - CHI'98, pages 496-502, New York, New York, USA, Jan. 1998. ACM Press.

[21] D. Stotts, J. Smith, and K. Gyllstrom. FaceSpace: Endo- and ExoSpatial Hypermedia in the Transparent Video Facetop. In HYPERTEXT '04, pages 48-57, 2004.

[22] A. Tang, J. Lanir, S. Greenberg, and S. Fels. Supporting transitions in work: informing large display application design by understanding whiteboard use. In GROUP '09: Conference on Supporting Group Work, pages 149-158, 2009.

[23] A. Tang, C. Neustaedter, and S. Greenberg. VideoArms: Embodiments for Mixed Presence Groupware, pages 85 - 102. Springer London, London, 2007.

[24] J. C. Tang. Findings from observational studies of collaborative work. International Journal of Man-Machine Studies, 34(2):143-160, Feb. 1991.

[25] J. C. Tang and S. Minneman. VideoWhiteboard: video shadows to support remote collaboration. In CHI '91: Proceedings of the SIGCHI conference on Human factors in computing systems, pages 315-322. ACM New York, 1991.

[26] J. C. Tang and S. L. Minneman. Videodraw: a video interface for collaborative drawing. ACM Transactions on Information Systems (TOIS), 9(2):170-184, 1991.

[27] M. Uflacker, T. Kowark, and A. Zeier. An Instrument for Real-Time Design Interaction Capture and Analysis. In C. Meinel, L. Leifer, and H. Plattner, editors, Design Thinking, Understand - Improve - Apply, pages 131-145. Springer Berlin Heidelberg, 2011.

[28] E. S. Veinott, J. S. Olson, G. M. Olson, and X. Fu. Video helps remote work: speakers who need to negotiate common ground benefit from seeing each other. In CHI '99: extended abstracts on Human factors in computing systems, pages 302-309, 1999.

[29] Y. Yoo and M. Alavi. Media and Group Cohesion: Relative Influences on Social Presence, Task Participation, and Group Consensus. MIS Quarterly, 25(3):371 - 390, 2001. 